\title{
STED microscopy reveals crystal colour centres with nanometric resolution
}

\author{
Eva Rittweger', Kyu Young Han', Scott E. Irvine`, Christian Eggeling and Stefan W. Hell^
}

Because they have spin states that can be optically polarized and detected, fluorescent nitrogen vacancies in diamond ${ }^{1-3}$ have considerable potential for applications in quantum cryptography $^{4,5}$ and computation ${ }^{6-8}$, as well as for nanoscale magnetic imaging ${ }^{9,10}$ and biolabelling ${ }^{11,12}$. However, their optical detection and control are hampered by the diffraction resolution barrier of far-field optics. Here, we show that stimulated emission depletion (STED) microscopy ${ }^{13,14}$ is capable of imaging nitrogen-vacancy centres with nanoscale resolution and Angström precision using focused light. The far-field optical control of the population of their excited state at the nanoscale expands the versatility of these centres and demonstrates the suitability of STED microscopy to image dense colour centres in crystals. Nitrogen-vacancy defects show great potential as tags for far-field optical nanoscopy ${ }^{15}$ because they exhibit nearly ideal STED without bleaching. Measured point-spread functions of $5.8 \mathrm{~nm}$ in width demonstrate an all-physics-based far-field optical resolving power exceeding the wavelength of light by two orders of magnitude.

For many decades, diffraction precluded far-field optical imaging with resolution better than half the wavelength of light. This limit has been overcome with the advent of STED microscopy, which is a lens-based fluorescence microscope with a resolution conceptually no longer limited by diffraction ${ }^{13,16,17}$. In brief, a STED microscope separates two or more adjacent objects by ensuring that only one of them fluoresces at a time. Sequential object signalling is accomplished by transiently switching the objects off by stimulated emission.

STED microscopy has so far provided a resolution of $16-80 \mathrm{~nm}$, which has been used for mapping proteins in cells ${ }^{18}$, and for the real-time imaging of colloidal particles or synaptic vesicles in living neurons ${ }^{19}$. The fluorescent markers used were organic molecules or fluorescent proteins having a singlet ground state $\left(S_{0}\right)$ and a first excited state $\left(S_{1}\right)$, each with vibrational sublevels. Once excited, these molecules equilibrate to the lowest vibrational sublevel of the $S_{1}$ state, from where they can return to a vibrational sublevel of the $S_{0}$ state by fluorescence emission after an average period $\tau_{\mathrm{fl}}$. Alternatively, the so-called STED beam switches the molecules off by forcing them down to the $S_{0}$ state. This occurs if the stimulated emission rate $\sigma I_{\mathrm{STED}} \lambda_{\mathrm{STED}} / h c$ is much larger than that of the spontaneous decay, $1 / \tau_{\mathrm{fl}}$, where $\sigma$ is the cross-section for stimulated emission, $h$ is Planck's constant, $c$ is the speed of light in vacuum, and $I_{\mathrm{STED}}$ is the STED beam intensity. The wavelength $\lambda_{\mathrm{STED}}$ is chosen such that the fluorophore is pumped into a rapidly decaying higher vibrational sublevel of the $S_{0}$ state, which makes de-excitation predominant. If we neglect polarization, the ability of the molecule to fluoresce decreases in proportion to $\exp \left(-I_{\mathrm{STED}} / I_{\mathrm{S}}\right)$ where $I_{\mathrm{S}}$ is a fluorophore characteristic $^{17}$. Hence, for $I_{\text {STED }} \geq 3 I_{\mathrm{S}}$ the fluorophore is essentially switched off.

Organic fluorophores follow such switching, but have the disadvantage of suffering from bleaching and blinking, making
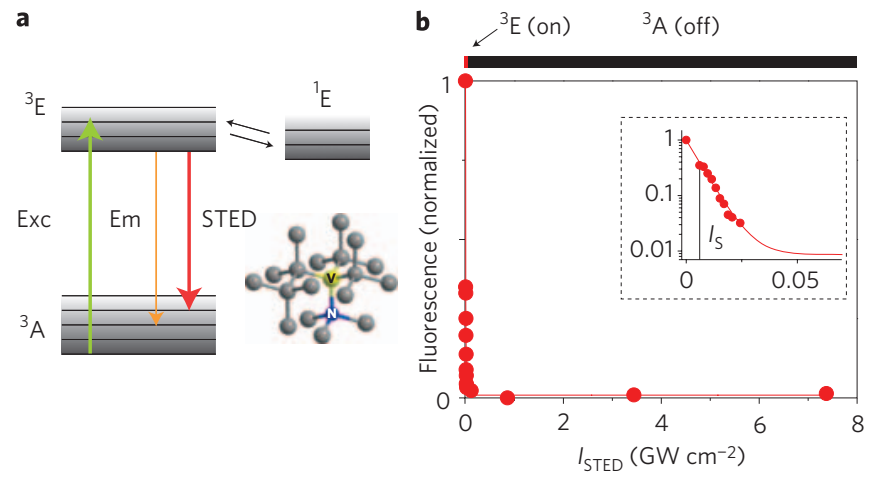

Figure 1 | Stimulated emission depletion of the excited state 'switches off' a charged nitrogen-vacancy colour centre by light. a, Energy diagram sketching the ground $\left({ }^{3} \mathrm{~A}\right)$, fluorescent $\left({ }^{3} \mathrm{E}\right)$ and the dark singlet state $\left({ }^{1} \mathrm{E}\right)$ along with excitation (Exc), emission (Em) and stimulated emission (STED) transitions in nitrogen-vacancy centres. $\mathbf{b}$, The steep ('rectangular')

fluorescence depletion curve showing the abrupt decrease of the population of ${ }^{3} \mathrm{E}$ with intensity $I_{\text {STED }}$ facilitates a nearly perfect implementation of STED. The inset representation confirms the exponential suppression of the excited state. For $I_{\text {STED }}>20 \mathrm{MW} \mathrm{cm}^{-2}$, the centre is deprived of its ability to fluoresce (switched off).

solid-state-based fluorophores very appealing. It is also interesting, however, to explore applications of far-field optical nanoscopy to transparent solids. Luminescent transitions arising from defects in diamond, particularly nitrogen-vacancy centres composed of a substitution nitrogen next to a charged vacancy (Fig. 1a), have attracted much attention ${ }^{2,20}$. Absorption at $532 \mathrm{~nm}$ elevates them from their ground $\left({ }^{3} \mathrm{~A}\right)$ state to an excited $\left({ }^{3} \mathrm{E}\right)$ state of $\tau_{\mathrm{fl}}=11.6 \mathrm{~ns}$ lifetime, which emits at $600-850 \mathrm{~nm}$ with virtually no bleaching or blinking. Phononic coupling yields a pronounced Stokes shift, but the involved states are now spin triplets. Centres with magnetic quantum number $m_{\mathrm{s}}=0$ emit more strongly, because their $m_{\mathrm{s}}= \pm 1$ counterparts have an increased tendency to convert into a dark singlet state $\left({ }^{1} \mathrm{E}\right)$, ultimately yielding ground-state centres with $m_{\mathrm{s}}=0$, a feature that allows for the orientation and detection of spin by illumination ${ }^{2,20}$. When used as scanning tips, nitrogenvacancy centres in nanosized diamonds provide optical ${ }^{21}$ and magnetic imaging ${ }^{9,10}$, but the prospect of reading and controlling individual defects at the nanoscale with conventional lenses is very attractive.

We therefore investigated STED on nitrogen-vacancy defects within diamonds of type IIa grown by chemical vapour deposition. Excitation at $532 \mathrm{~nm}$ was achieved using a 60-ps pulsed laser diode, which was triggered by the pulses of the STED beam provided by a laser system operating at $\lambda_{\mathrm{STED}}=775 \pm 1 \mathrm{~nm}$ and emitting $3.2 \mathrm{~ns}$ pulses at $8 \mathrm{MHz}$. The synchronized pulses were combined using 

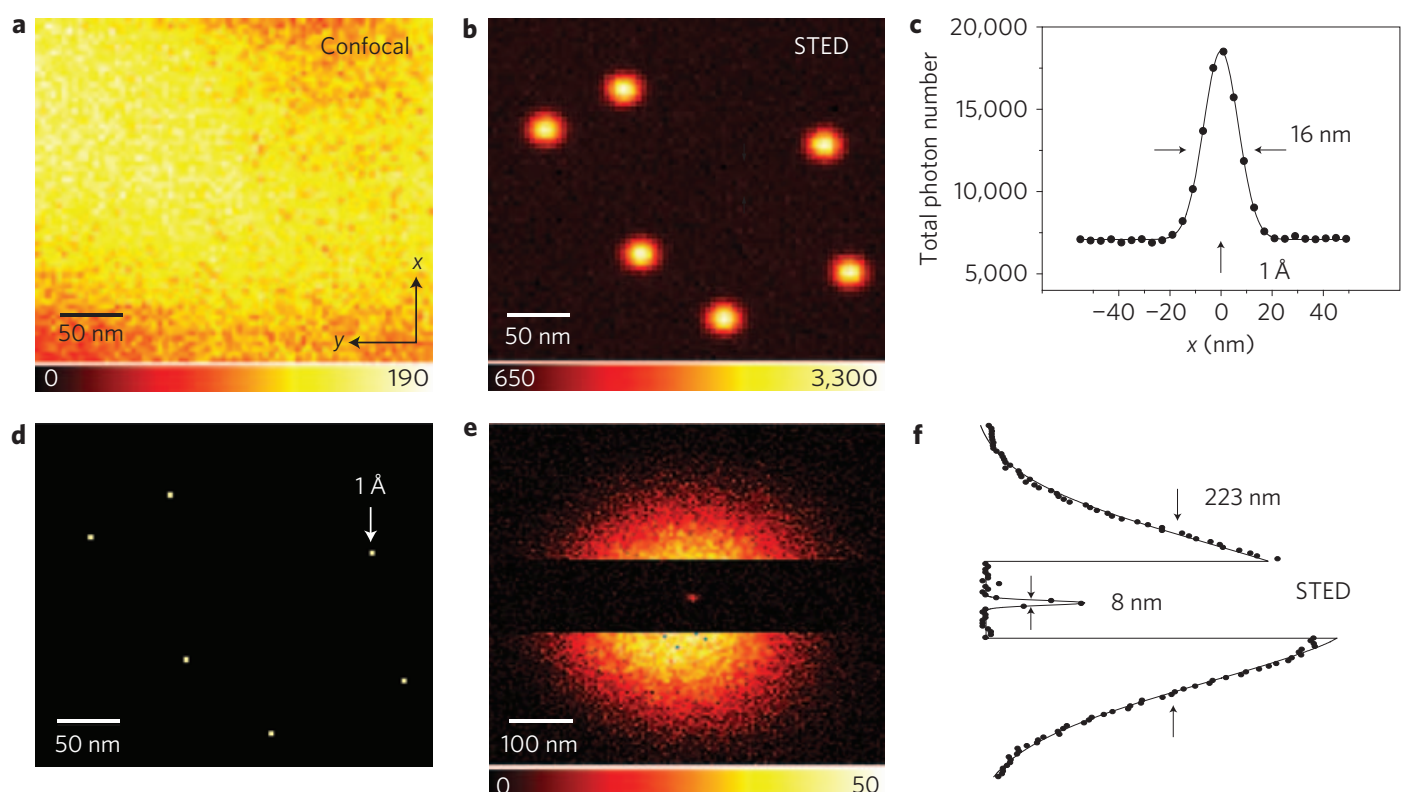

Figure 2 | Stimulated emission depletion microscopy reveals densely packed nitrogen-vacancy centres in diamond. a,b, Confocal (a) and STED (b) images from the same crystal region. $\mathbf{c}$, The individual centres resolved in $\mathbf{b}$ automatically yield the effective PSF of the STED recording whose $y$-profile exhibits a FWHM of $\Delta y=16.0 \mathrm{~nm}$. d, The coordinate of each centre can be calculated with $0.14 \mathrm{~nm}$ precision. Comparing a with $\mathbf{d}$ highlights the dramatic

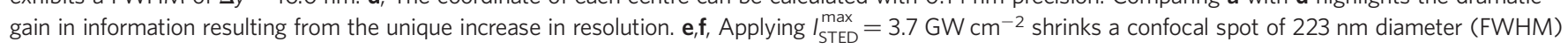
down to $8 \mathrm{~nm}$. Note that the increase in resolution is a purely physical phenomenon.

dichroic mirrors and collinearly directed through a 1.46 numerical aperture (NA) oil immersion lens, which also imaged the fluorescence onto the confocally arranged aperture of a counting avalanche photodiode.

The phononic coupling in the ${ }^{3} \mathrm{~A}$ state enables quenching of excited nitrogen-vacancy centres by stimulated emission. This is demonstrated in Fig. 1b, which shows the fluorescence of a single centre as a function of $I_{\mathrm{STED}}$, here defined as the peak intensity in the focal plane. The time-averaged power of the excitation light was $40 \mu \mathrm{W}$. A steep exponential drop of the fluorescence with $I_{\text {STED }}$ is observed, as one would anticipate for perfect STED (ref. 13). Extracting $I_{\mathrm{S}}=6.6 \mathrm{MW} \mathrm{cm}{ }^{-2}$ yields $\sigma=1.2 \times 10^{-17} \mathrm{~cm}^{2}$, which agrees with the value determined from the emission spectrum. The main outcome, however, is that the nitrogen-vacancy centres can be sharply switched off by light (Fig. 1b).

To make use of this nearly 'rectangular' depletion curve ${ }^{13}$ for nanoscopy, we produced a doughnut-shaped focal intensity distribution $^{22} I_{\mathrm{STED}}^{\mathrm{d}}(x, y)$ with a central minimum $I_{\mathrm{STED}}^{\min }(0,0) \approx 0$. The minimum is ideally zero, but in practice it amounts to a fraction $\varepsilon \ll 1$ of the maximum intensity at the doughnut crest: $I_{\mathrm{STED}}^{\min }=\varepsilon \max \left[\left(I_{\mathrm{STED}}^{\mathrm{d}}(x, y)\right]\right.$. Overlapping the Airy disk of the excitation beam with the doughnut and enforcing $I_{\mathrm{STED}}^{\max } \gg I_{\mathrm{S}}$ switched off all the centres covered by the Airy disk except for those at the doughnut minimum where $I_{\mathrm{STED}}^{\mathrm{d}}(x, y) \leq 3 I_{\mathrm{S}}$. Therefore, the Airy disk can be ignored when calculating the spot in which the centres can still be 'on', that is, the effective point-spread function (PSF) of the system. It is described by the function $\exp \left(-I_{\mathrm{STED}}^{\mathrm{d}}(x, y) / I_{\mathrm{S}}\right)$ having a full-width at half-maximum (FWHM) of $\Delta x, y \approx \lambda_{\mathrm{STED}} / \sqrt{ }\left(C I_{\mathrm{STED}}^{\max } / I_{\mathrm{S}}\right)$, with the constant $C$ depending on the shape of the minimum ${ }^{22}$. Although the resolution $\Delta x, y$ scales with the wavelength, adjusting $I_{\mathrm{STED}}^{\max } \gg I_{\mathrm{S}}$ squeezes the PSF continuously, indicating that the wavelength has surrendered its limiting role.

We imaged by scanning the sample with respect to the beams using a three-dimensional piezo stage (Physik Instrumente, P-733.3DD) with $0.1 \mathrm{~nm}$ positional accuracy and $0.03 \%$ linearity. Figure 2 compares a confocal image (Fig. 2a) with the corresponding STED image
(Fig. 2b), taken at $I_{\mathrm{STED}}^{\max }=1.4 \mathrm{GW} \mathrm{cm}^{-2}$. Although the confocal reference lacks any detail, its STED counterpart resolves each nitrogen-vacancy defect. The similar brightness and sparse occurrence of the dots shown in the crystal allow us to conclude that they represent single nitrogen-vacancy defects. The images consist of $75 \times 65$ pixels of $4 \times 4 \mathrm{~nm}$ in size, recorded with a dwell time of $5 \mathrm{~ms}$. To reduce statistical error (number of counts given in the colour table), the displayed and analysed STED image comprises the sum of 97 consecutively recorded images. Representing the effective PSF, the images of individual centres reveal $\Delta x=18.3 \mathrm{~nm}$ and $\Delta y=16.0 \mathrm{~nm}$ as the resolution under the conditions used. Note that the resolution gain derives just from the optical preparation of the states.

The STED recording inherently resolves individual nitrogenvacancy centres because they are all further apart from each other than $\Delta x$ or $\Delta y$. An important insight is that, when scanned over the sample together with the excitation beam, the doughnutshaped STED beam allows only a single nitrogen-vacancy centre to fall within the doughnut minimum; all others are switched off or simply not excited. Thus STED nitrogen-vacancy centres are resolved individually and hence registered sequentially in time.

Once all the centres are resolved, increasing the resolution further is no longer required. Their position can be calculated from the centroid of their subdiffraction-sized $\operatorname{spot}^{23}$. The precision of this calculation depends on the effective PSF, the number of collected photons and the background ${ }^{24,25}$. We measured a spatially homogeneous background of 1.6 counts $\mathrm{ms}^{-1}$ throughout the imaged region, as indicated in the images. Under these conditions and the assumption (here not made or asserted) that scanning errors are negligible, the $x y$-coordinates of each centre can be derived with 1-2 A precision; that is, by three orders of magnitude below the diffraction limit (Fig. 2d). The information provided in Fig. $2 \mathrm{~d}$ should be contrasted with that contained in the confocal recording (Fig. 2a), which fails to render any information about the number and location of the colour centres. The distance between the centres in the upper left corner projected in the $x y$ plane is $67.9 \pm 0.1 \mathrm{~nm}$. By extending STED into the third dimension, one can also incorporate depth information. Thus, provided 

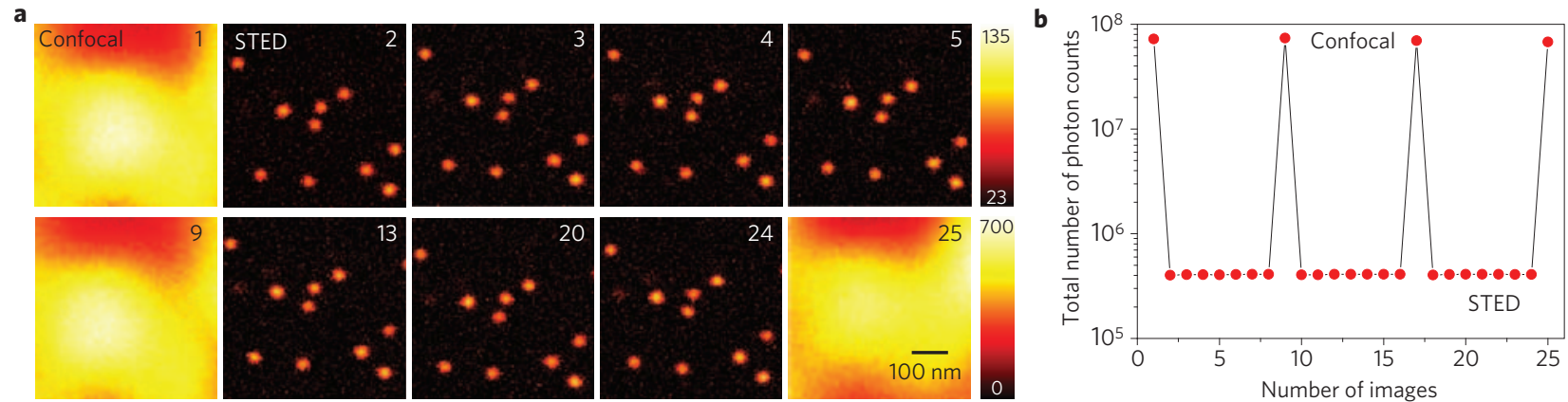

Figure 3 | STED microscopy without photobleaching. a, Consecutive confocal and STED recordings (as indicated) of the same crystal region. $\mathbf{b}$, The total fluorescence signal in each image frame, showing virtually no change in brightness and in resolution (here $<25 \mathrm{~nm}$ ) with increasing number of recordings. The recording time for all images was the same, causing the STED images to be darker approximately by the (here $\sim 100$-fold) reduction in focal area. Importantly, the peak brightness of single centres is virtually equal in the STED and confocal recordings. Note the absence of blinking. The experiment demonstrates far-field optical nanoscopy recording without photobleaching.
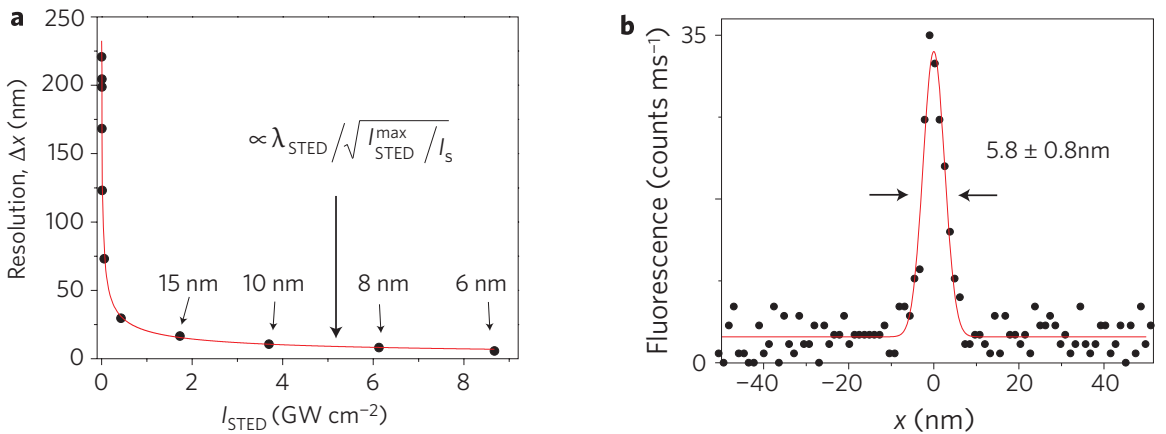

Figure 4 | Maximizing the resolving power by optimizing STED in a single direction. a, The measured decrease of the lateral FWHM $\Delta x$ from $223 \mathrm{~nm}$ down to $6 \mathrm{~nm}$ scales inversely with the square-root of the intensity of the STED beam (red line). Note that all data were taken from the same single nitrogenvacancy defect. $\mathbf{b}$, A line profile of the effective PSF along the $x$-axis, demonstrating an unprecedented all-physics-based far-field optical resolving power of $5.8 \pm 0.8 \mathrm{~nm}$.

that care is exerted to avoid scanning distortions and drift, STED microscopy is able to map nitrogen-vacancy centres with nanometric resolution and subnanometre precision.

Figure 2e shows a confocal image of an isolated nitrogen-vacancy centre, but near its middle, the STED beam is momentarily engaged. Applying $I_{\mathrm{STED}}^{\max }=3.7 \mathrm{GW} \mathrm{cm} \mathrm{cm}^{-2}$ produced a spot of $8 \mathrm{~nm}$ FWHM. In this case, the peak is also reduced by 3.5 -fold, which is due to the nonzero doughnut minimum $I_{\mathrm{STED}}^{\min }(0,0)=\varepsilon I_{\mathrm{STED}}^{\max }$ suppressing the peak by $\exp \left(-\varepsilon I_{\mathrm{STED}}^{\max } / I_{\mathrm{S}}\right)$. Clearly, such peak reductions become important at large $I_{\text {STED }}^{\max }$ and therefore at high resolution. Evaluation of the measurement using Fig. $1 \mathrm{~b}$ yields $\varepsilon=7 \times 10^{-4}$, which is due to doughnut imperfections. Note that the effective focal area decreases by a factor of 777 .

Importantly, the outstanding photostability of nitrogen-vacancy centres $^{1}$ is maintained under the intense STED beams, as made evident in consecutive STED recordings (Fig. 3) in which both the brightness and the tenfold resolution increase remain unchanged. Demonstrating optical nanoscopy without photobleaching, Fig. 3 highlights that with diamond nanoparticles as markers $^{11,12}$, STED will enable the use of (biological) fluorescence nanoscopy with countless recordings.

Recording images similar to those in Figs 2 and 3 is impractical with near-field optics, because, being located more than $2-3 \mu \mathrm{m}$ beneath the surface of the crystal, the colour centres cannot be accessed by a tip. Besides being noninvasive and able to access the interior of the crystal, compared to near-field optics, STED microscopy is easier to put into practice, control and operate, and provides substantially higher resolution. Likewise, alternative nanoscopy strategies that sparsely switch individual emitters (from a dark state to a state emitting a bunch of photons allowing their localization $^{26-29}$ ) are hard to implement for nitrogen-vacancy colour centres, because at room temperature, blinking or metastable switching between a bright and a dark state is nearly absent. Hence, nitrogen-vacancy centres are ideal for STED and STED is ideal for their imaging. This is likely to apply to many other colour centres in crystals.

Next we used a focal STED beam spot with a $y$-oriented central zero-line with steep peaks $I_{\text {STED }}^{\max }$ along the $x$-axis ${ }^{16}$. Increasing $I_{\text {STED }}^{\max }$ to $8.6 \mathrm{GW} \mathrm{cm} \mathrm{cm}^{-2}$ squeezes the FWHM of the PSF from $\Delta x=223 \mathrm{~nm}$ down to $6 \mathrm{~nm}$ (Fig. $4 \mathrm{a}$ ). The increase in resolution follows the anticipated inverse square-root law ${ }^{15,16}$. An exemplary super-resolved PSF of a single nitrogen-vacancy centre is shown in Fig. $4 \mathrm{~b}$ and has a measured $\Delta x=5.8 \pm 0.8 \mathrm{~nm}$. This value is 133 -fold below the responsible wavelength and represents a new regime of obtainable all-physics-based resolution using conventional optics.

Despite the leaps and bounds STED microscopy has made, its limits have not yet been reached. Applying a shorter $\lambda_{\text {STED }}$ entailing a larger $\sigma$ should decrease $\Delta x, \Delta y$ even further. Implemented with a deep zero $\left(\varepsilon \ll 1 \times 10^{-4}\right)$, the initially desired nearly 'rectangular' depletion curve $^{13}$ should enable a further 'spot sharpening' by more than a factor of 10 , which may surpass the dimensions of the electron cloud and, leaving the dipole approximation behind, open up the fascinating prospect of sensing the (size of the) cloud and its immediate environment. It would also be desirable to investigate STED on nitrogen-vacancy centres subject to magnetic and microwave fields. In particular, reading out nitrogen-vacancy centres and hence local magnetic fields on the nanoscale should be easier 
with STED and conventional lenses than with nanosized optical tips. Thus, STED microscopy may greatly simplify magnetic imaging at the nanoscale $e^{9,10}$. Likewise it will be interesting to apply STED nanoscopy to other colour centres and crystals. Finally, the achieved and future gains in all-physics-based resolution should stimulate novel explorations in applied and fundamental (quantum) optics.

\section{Methods}

The cylindrical doughnut focal spot used for generating the data of Figs 2 and 3 was produced by inserting a glass waveplate (RPC Photonics), inducing an azimuthal linear helical phase ramp from 0 to $2 \pi$ on the initially flat wavefront of the STED beam. In these experiments both the excitation and the STED beam were circularly polarized. The bandwidth of the STED beam was $<10 \mathrm{GHz}$.

The data of Fig. 4 were produced with linearly polarized excitation and STED beams. A phase shift of $\pi$, resulting in the $y$-oriented line-shaped valley, was produced by inserting a home-built phase-shifter consisting of tilted optical flats. In this case, the polarization of both beams was oriented along the direction of the lineshaped valley. Images in Fig. 2a,b were taken from a diamond sample with a higher density of nitrogen-vacancy centres than the crystal used for the measurements shown in Figs 2e and 4a,b.

The intensity values refer to spatial and temporal peak intensities assuming aberration-free focusing and rectangular laser pulses, with the transmission of the objective lens $70 \%$ at $775 \mathrm{~nm}$. A 95\% transmission was assumed at the interface between the immersion oil and the diamond crystal. In all recordings, background noise was reduced to 120 counts s ${ }^{-1}$ by gated detecting within a 40 -ns window after the STED pulse.

The stimulated emission cross-section was calculated using $\sigma \equiv h c /\left(\lambda_{\mathrm{STED}} I_{\mathrm{S}} \tau_{\mathrm{p}}\right)$ under the approximation that the change in population of the excited state of the nitrogen-vacancy centre was negligible during the duration $\tau_{\mathrm{p}}$ of the STED pulse. The cross-section was also derived from the emission spectrum $E(\lambda)$ of a single defect normalized to the quantum yield ${ }^{3,30}\left(Q=0.7=\int E(\lambda) \mathrm{d} \lambda\right)$ using $\sigma=\lambda^{4} E(\lambda) / 8 \pi c n^{2} \tau_{\mathrm{fl}}$, with the speed of light in vacuum $c$ and the refractive index of diamond $n=2.417$.

The STED image of Fig. $2 b$ was generated by summing 97 STED images of the same region. Alignment was ensured by introducing subpixel translational shifts to maximize the spatial correlation between the images. This corrects exclusively translational drift between consecutive images. The central positions of the defects in the registered images were stochastically distributed with a variance close to the expected localization accuracy, supporting the notion that no further substantial corrections were required in this case. The centroids were determined by Gaussian fits.

Data points within Fig. 4a were fitted with the general expression ${ }^{22}$ $\Delta x \approx x_{\mathrm{c}} / \sqrt{ }\left(1+x_{\mathrm{c}}^{2} a^{2} I_{\mathrm{STED}}^{\max } /\left(I_{\mathrm{S}} \ln 2\right)\right)$, where $x_{\mathrm{c}}$ is the FWHM for $I_{\mathrm{STED}}^{\max }=0$ and $a$ is the steepness of the STED intensity pattern $I_{\mathrm{STED}}(x) \equiv 4 I_{\mathrm{STED}}^{\max } a^{2} x^{2}$, giving $a=1.76 \lambda / \mathrm{NA}$. The measured STED PSF independently yielded a steepness $a=1.75 \lambda / \mathrm{NA}$. The line profile of Fig. $4 \mathrm{~b}$ represents eight consecutive $x$-scans over the same nitrogen-vacancy defect, revealing an average measured FWHM of the scans of $5.8 \mathrm{~nm}$, with a standard deviation of $0.8 \mathrm{~nm}$.

\section{Received 6 November 2008; accepted 20 January 2009; published online 22 February 2009}

\section{References}

1. Gruber, A. et al. Scanning confocal optical microscopy and magnetic resonance on single defect centers. Science 276, 2012 -2014 (1997).

2. Jelezko, F. \& Wrachtrup, J. Single defect centres in diamond: a review. Phys. Status Solidi A 203, 3207-3225 (2006).

3. Wrachtrup, J. \& Jelezko, F. Processing quantum information in diamond. J. Phys. Condens. Matter 18, S807-S824 (2006).

4. Kurtsiefer, C., Mayer, S., Zarda, P. \& Weinfurter, H. Stable solid-state source of single photons. Phys. Rev. Lett. 85, 290-293 (2000).

5. Beveratos, A. et al. Single photon quantum cryptography. Phys. Rev. Lett. 89, 187901 (2002).

6. Gaebel, T. et al. Room-temperature coherent coupling of single spins in diamond. Nature Phys. 2, $408-413$ (2006).

7. Gurudev Dutt, M. V. et al. Quantum register based on individual electronic and nuclear spin qubits in diamond. Science 316, $1312-1316$ (2007).
8. Hanson, R., Dobrovitski, V. V., Feiguin, A. E., Gwyat, O. \& Awschalom, D. D. Coherent dynamics of a single spin interacting with an adjustable spin bath. Science 320, 352 - 355 (2008).

9. Balasubramanian, G. et al. Nanoscale imaging magnetometry with diamond spins under ambient conditions. Nature 455, 648-651 (2008).

10. Maze, J. R. et al. Nanoscale magnetic sensing with an individual electronic spin in diamond. Nature 455, 644-647 (2008).

11. Fu, C. C. et al. Characterization and application of single fluorescent nanodiamonds as cellular biomarkers. Proc. Natl Acad. Sci. USA 104, $727-732$ (2007).

12. Chao, J. I. et al. Nanometer-sized diamond particle as a probe for biolabeling. Biophys. J. 93, 2199-2208 (2007).

13. Hell, S. W. \& Wichmann, J. Breaking the diffraction resolution limit by stimulated emission: stimulated emission depletion fluorescence microscopy. Opt. Lett. 19, 780-782 (1994).

14. Klar, T. A. \& Hell, S. W. Subdiffraction resolution in far-field fluorescence microscopy. Opt. Lett. 24, 954-956 (1999)

15. Hell, S. W. Toward fluorescence nanoscopy. Nature Biotechnol. 21, $1347-1355$ (2003).

16. Westphal, V. \& Hell, S. W. Nanoscale resolution in the focal plane of an optical microscope. Phys. Rev. Lett. 94, 143903 (2005).

17. Dyba, M. \& Hell, S. W. Focal spots of size $\lambda / 23$ open up far-field fluorescence microscopy at $33 \mathrm{~nm}$ axial resolution. Phys. Rev. Lett. 88, 163901 (2002).

18. Donnert, G. et al. Macromolecular-scale resolution in biological fluorescence microscopy. Proc. Natl Acad. Sci. USA 103, $11440-11445$ (2006)

19. Westphal, V. et al. Video-rate far-field optical nanoscopy dissects synaptic vesicle movement. Science 320, 246-249 (2008)

20. Manson, N. B., Harrison, J. P. \& Sellars, M. J. Nitrogen-vacancy center in diamond: Model of the electronic structure and associated dynamics. Phys. Rev. B 74, 104303 (2006).

21. Kühn, S., Hettich, C., Schmitt, C., Poizat, J. P. H. \& Sandoghdar, V. Diamond colour centres as a nanoscopic light source for scanning near-field optical microscopy. J. Microsc. 202, 2-6 (2001).

22. Harke, B. et al. Resolution scaling in STED microscopy. Opt. Express 16, 4154-4162 (2008).

23. Heisenberg, W. The Physical Principles of the Quantum Theory (Chicago Univ. Press, 1930).

24. Bobroff, N. Position measurement with a resolution and noise-limited instrument. Rev. Sci. Instrum. 57, $1152-1157$ (1986).

25. Yildiz, A. et al. Myosin V walks hand-over-hand: single fluorophore imaging with 1.5-nm localization. Science 300, $2061-2065$ (2003).

26. Gordon, M. P., Ha, T. \& Selvin, P. R. Single-molecule high-resolution imaging with photobleaching. Proc. Natl Acad. Sci. USA 101, $6462-6465$ (2004).

27. Betzig, E. et al. Imaging intracellular fluorescent proteins at nanometer resolution. Science 313, $1642-1645$ (2006).

28. Rust, M. J., Bates, M. \& Zhuang, X. Sub-diffraction-limit imaging by stochastic optical reconstruction microscopy (STORM). Nature Meth. 3, $793-796$ (2006).

29. Fölling, J. et al. Fluorescence nanoscopy by ground-state depletion and singlemolecule return. Nature Meth. 5, $943-945$ (2008).

30. Rittweger, E., Rankin, B. R., Westphal, V. \& Hell, S. W. Fluorescence depletion mechanisms in super-resolving STED microscopy. Chem. Phys. Lett. 442, $483-487$ (2007)

\section{Acknowledgements}

We acknowledge motivating discussions with R. Walsworth and M. Lukin about magnetic imaging and also with F. Jelezko, who provided us with the nitrogen-vacancy diamond crystals. Furthermore, we thank A. Schönle for support with the software ImSpector, and J. Keller and M. Lauterbach for help with data analysis software. S.E.I. and K.Y.H gratefully acknowledge support from the Natural Sciences and Engineering Research Council of Canada and from the Korea Research Foundation Grant funded by the Korean Government (MOEHRD), respectively. K.Y.H. is on leave from the Department of Chemistry, Seoul National University, Korea.

\section{Additional information}

Reprints and permission information is available online at http://npg.nature.com/ reprintsandpermissions/. Correspondence and requests for materials should be addressed to S.W.H. 